1. Akiyama, H., et al. 2000. Inflammation and Alzheimer's disease. Neurobiol. Aging. 21:383-421.

2. Wyss-Coray, T., and Mucke, L. 2002. Inflammation in neurodegenerative disease--a double-edged sword. Neuron. 35:419-432.

3. Glabe, C.C. 2005. Amyloid accumulation and pathogensis of Alzheimer's disease: significance of monomeric, oligomeric and fibrillar Abeta. Subcell. Biochem. 38:167-177.

4. Kayed, R., et al. 2003. Common structure of soluble amyloid oligomers implies common mechanism of pathogenesis. Science. 300:486-489.

5. Walsh, D.M., et al. 2002. Naturally secreted oligomers of amyloid beta protein potently inhibit hippocampal long-term potentiation in vivo. Nature. 416:535-539.

6. Lesne, S., et al. 2006. A specific amyloid-beta protein assembly in the brain impairs memory. Nature. 440:352-357

7. Billings, L.M., Oddo, S., Green, K.N., McGaugh, J.L., and Laferla, F.M. 2005. Intraneuronal Abeta causes the onset of early Alzheimer's disease-relat- ed cognitive deficits in transgenic mice. Neuron. 45:675-688.

8. Tesseur, I., et al. 2006. Deficiency in neuronal TGF- $\beta$ signaling promotes neurodegeneration and Alzheimer's pathology.J. Clin. Invest. 116:3060-3069. doi:10.1172/JCI27341.

9. Unsicker, K., and Krieglstein, K. 2002. TGF-betas and their roles in the regulation of neuron survival. Adv. Exp. Med. Biol. 513:353-374.

10. Vivien, D., et al. 1998. Evidence of type I and type II transforming growth factor-beta receptors in central nervous tissues: changes induced by focal cerebral ischemia. J. Neurochem. 70:2296-2304

11. Han, G., et al. 2005. Distinct mechanisms of TGF- $\beta 1$ mediated epithelial-to-mesenchymal transition and metastasis during skin carcinogenesis. J. Clin. Invest. 115:1714-1723. doi:10.1172/JCI24399.

12. Gebken, J., et al. 1999. Ligand-induced downregulation of receptors for TGF-beta in human osteoblast-like cells from adult donors. J. Endocrinol. 161:503-510.

13. Li, M.O., Wan, Y.Y., Sanjabi, S., Robertson, A.K., and Flavell, R.A. 2006. Transforming growth factor-beta regulation of immune responses. Annu. Rev. Immunol. 24:99-146.

14. Liu, Y., Teige, I., Birnir, B., and Issazadeh-Navikas, S. 2006. Neuron-mediated generation of regulatory $T$ cells from encephalitogenic T cells suppresses EAE. Nat. Med. 12:518-525.

15. Farkas, L.M., Dunker, N., Roussa, E., Unsicker, K., and Krieglstein, K. 2003. Transforming growth factor-beta(s) are essential for the development of midbrain dopaminergic neurons in vitro and in vivo. J. Neurosci. 23:5178-5186.

16. Wyss-Coray, T., et al. 2001. TGF-beta1 promotes microglial amyloid-beta clearance and reduces plaque burden in transgenic mice. Nat. Med. 7:612-618.

17. Syken, J., Grandpre, T., Kanold, P.O., and Shatz, C.J. 2006. PirB restricts ocular-dominance plasticity in visual cortex. Science. In press.

18. Wyss-Coray, T., et al. 2002. Prominent neurodegeneration and increased plaque formation in complement-inhibited Alzheimer's mice. Proc. Natl. Acad. Sci. U. S. A. 99:10837-10842.

\title{
Microglia: a cellular vehicle for CNS gene therapy
}

\section{Harald Neumann}

Neural Regeneration Unit, Institute of Reconstructive Neurobiology, University of Bonn, and LIFE \& BRAIN Center and Hertie Foundation, Bonn, Germany.

\begin{abstract}
Metachromatic leukodystrophy (MLD) is a lysosomal storage disease caused by deficiency of the enzyme arylsulfatase A (ARSA). MLD is characterized by progressive demyelination and neurological deficits. Treatment of MLD is still a challenge due to the fact that the blood-brain barrier is a major obstacle for most therapeutic substances. In this issue of the JCI, Biffi et al. report that genetically modified hematopoietic precursor cells transduced to overexpress ARSA and transplanted into mice with a targeted disruption of the murine Arsa gene (Arsa-/- mice) migrated into the CNS and crosscorrected brain ARSA deficiency (see the related article beginning on page 3070). Microglia served as a cellular vehicle to effectively deliver the enzyme to other brain cells while hepatocytes overexpressing ARSA increased plasma ARSA levels but failed to deliver ARSA into the CNS.
\end{abstract}

\section{The blood-brain barrier: an obstacle for CNS therapy}

Innate and adaptive immune responses are strongly reduced in the CNS, leading to a status of immune privilege. This is a highly regulated and adapted process in which those immune responses that would induce collateral injury to "innocent bystander" tissues are avoided while certain features of the immune response are allowed (1). This special type of immune compromise protects CNS tissue, particularly neurons and their connections, from immune-mediated damage that would threaten the survival of the host.

Nonstandard abbreviations used: ARSA, arylsulfatase A; BBB, blood-brain barrier; LSD, lysosomal storage disorder; MLD, metachromatic leukodystrophy.

Conflict of interest: The author has declared that no conflict of interest exists.

Citation for this article: J. Clin. Invest. 116:2857-2860 (2006). doi:10.1172/JCI30230.
The blood-brain barrier (BBB) strongly contributes to the immune-privileged status of the CNS. The BBB is a unique membranous barrier that tightly segregates the brain from the circulating blood. The capillaries of the CNS form a permeability barrier and are structurally different from other tissues (Figure 1). The blood capillaries of the vertebrate brain and spinal cord are lined with a layer of special endothelial cells that lack fenestrations and are sealed with tight junctions. The tight junctions between endothelial cells result in a very high transendothelial electrical resistance of $1500-2000 \Omega \mathrm{cm}^{2}$ in the CNS compared with $3-33 \Omega \mathrm{cm}^{2}$ in other tissues $(2,3)$. Furthermore, astrocyte foot processes encapsulate the capillaries, forming a second barrier of the BBB. Therefore, only lipid-soluble molecules that can freely diffuse through the capillary endothelial membrane may passively cross the BBB. This bar- rier makes the CNS practically inaccessible to lipid-insoluble compounds such as small polar molecules or any larger protein.

Consequently, the BBB is an obstacle for CNS therapy and a serious bottleneck in drug development for CNS diseases. Theoretically, the amount of randomly selected drugs having bioavailability in the CNS is less than $2 \%$ of small molecules and practically $0 \%$ of large molecules $(2,3)$. These numbers are also reflected by the drugs currently available for CNS diseases. Of over 7,000 potential drugs in the comprehensive medicinal chemistry database, only $5 \%$ of all drugs treat the CNS (4). Interestingly, the typical CNS-active drug is very small in size with an average molecular mass of $0.36 \mathrm{kDa}$ and is used for treatment of a very limited number of diseases, such as affective disorders, schizophrenia, epilepsy, and chronic pain $(2,3)$. Furthermore, some drugs are obtainable that utilize the active transport system of the BBB. For example, L-dihydroxyphenylalanine (L-DOPA), a dopamine-replacement therapy for Parkinson disease patients, is a substrate for the BBB transporter L-amino acid transporter-1 (5). Once it crosses the BBB, L-DOPA is decarboxylated back to dopamine via aromatic amino acid decarboxylase. Thus, L-DOPA is an example of a prodrug that traverses the biological membranes of the BBB, not via lipid mediation, but via a carrier transport system. 
A Capillary alongside blood-brain barrier

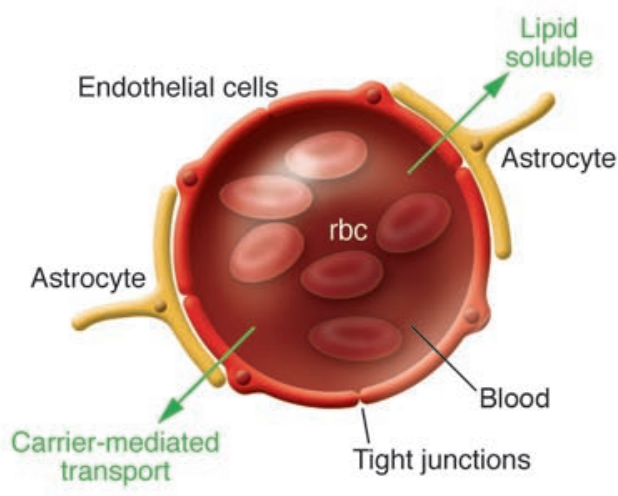

B Fenestrated or sinusoid capillary

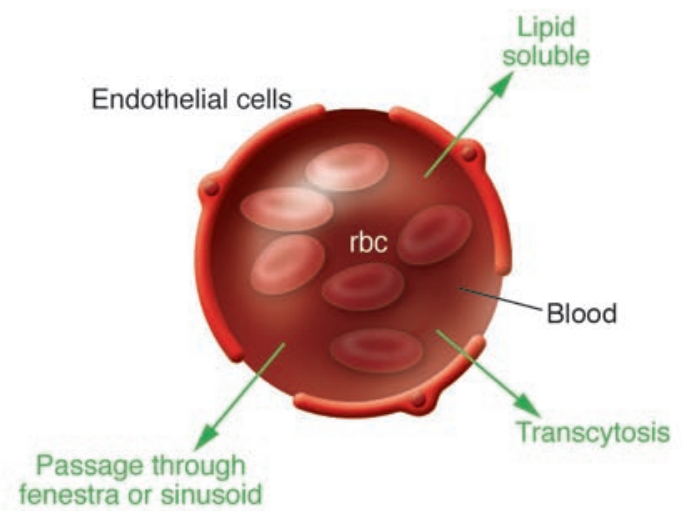

Figure 1

Comparison between the specialized capillaries of the CNS and those of other tissues. (A) Capillaries of the CNS are continuous capillaries. The endothelial cells have tight junctions and are covered by astrocytic end feet. Small lipophilic molecules can passively pass through the BBB. Furthermore, certain molecules can actively pass the BBB using the carrier-mediated cell membrane transport system. (B) Capillaries of other tissues are often fenestrated, moderately porous capillaries (renal glomeruli, intestinal villi, and in most glands) or sinusoid, highly porous capillaries (in the liver, bone marrow, lymphoid tissues, and some endocrine organs). These ultramicroscopic pores are responsible for the majority of exchange between the blood and tissue. Furthermore, capillary exchange is executed by transcytosis, a process in which the endothelial cell encloses material of the bloodstream in an invagination of the cell membrane to form a vesicle, then moves the vesicle across the endothelium cell to eject the material at the tissue side.

Interestingly, the BBB can easily be penetrated by certain types of immune cells, such as activated $T$ cells and macrophages, thereby offering a natural transport system for drugs or genes $(6,7)$. In this issue of the JCI, Biffi and colleagues report on their exploitation of this cellular transport system via the BBB to deliver gene therapy to mice with metachromatic leukodystrophy (MLD) (8).

\section{Hematopoietic origin of microglia}

The function of resident macrophages in the CNS is executed by microglial cells. Microglial cells are of hematopoietic origin and belong to the myelomonocytic lineage. They are derived from the hemangioblastic mesoderm and populate the developing neuroectoderm in rodents at embryonic day 8 (9). Microglial cells are quite numerous, and it is estimated that the rodent brain contains at least $10 \%$ microglial cells (9). In adulthood, undefined bone marrowderived myeloid precursor cells migrate into the CNS and become resident perivascular macrophages and occasionally microglia $(6,10,11)$. Thus, a small subpopulation of perivascular macrophages and microglial cells are continuously (and for the life span of the host) replenished by bone marrowderived cells. Interestingly, the bone marrow-derived myeloid precursor cells are preferentially recruited to sites of neuronal degeneration (6). Parenchymal microglial cells have been reported to be phenotypically very similar to uncommitted myeloid precursors, having "empty" MHC class II molecules, comparable cysteine protease profiles, and the capacity to differentiate into dendritic cell-like cells (12). The microglial cells have multiple long processes, which interdigitate the parenchymal nervous tissue and show high constitutive motility (13). Thus, microglial cells, which cover almost the entire CNS with their processes, might serve as an ideal cell type to mediate cross-correction of molecules that are lacking in neighboring brain cells.

\section{CNS gene therapy of MLD by genetically modified microglial precursors}

There is an obvious discrepancy between the tremendous progress made in molecular neurosciences and the treatment options available for CNS diseases. So far, there is no effective drug therapy for most lysosomal storage disorders (LSDs), although in many of these cases, the gene underlying the disease is known. LSDs are a group of more than 40 genetic diseases that are caused by the deficiency of 1 or more lysosomal proteins. The incidence of LSDs is estimated to be approximately 1 in 7,500 live births. The loss in enzymatic activity leads to the accumulation of nondegraded substrates within lysosomes, resulting in distension of the organelle and subsequent cellular malfunction. Gene therapy is being considered as a suitable treatment approach for this group of disorders.
The goal of gene therapy for LSDs is to introduce the DNA for the deficient lysosomal enzyme or protein into a cell, which would ideally lead to the sustained production and reconstitution of therapeutic levels of the protein in affected tissues. In vivo and ex vivo gene therapy approaches have been studied experimentally. The concept of ex vivo gene therapy of LSDs is based on cross-correction (Figure 2). Successful treatment depends on the transfer of the protein from the genetically modified donor cells to the protein-deficient recipient cells.

The LSD known as MLD is caused by the deficiency of arylsulfatase A (ARSA), a soluble lysosomal enzyme, which is involved in the degradation of acidic sphingolipid galactosylceramide $\mathrm{I}^{3}$-sulfate (sulfatide). The deficiency of this enzyme results in the intracellular accumulation of sulfatide in lysosomes. Sulfatide has an essential function in oligodendrocytes, supporting the correct organization of the myelin sheaths throughout the CNS. Accumulation of sulfatide in the lysosomal compartment of oligodendrocytes leads to cellular dysfunction and demyelination. Accordingly, MLD patients suffer from multiple neurological deficits due to progressive demyelination followed by axonal degeneration. It has been known for many years that a fraction of newly synthesized ARSA is secreted from many cell types, including immune cells. The oligosaccharide side chains of the secreted enzyme can bear mannose 6-phos- 
A Correction by in vivo gene therapy

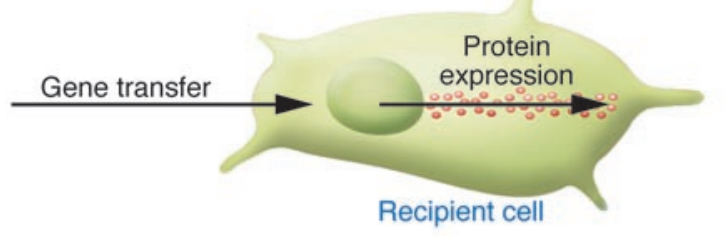

B Cross-correction by ex vivo gene therapy

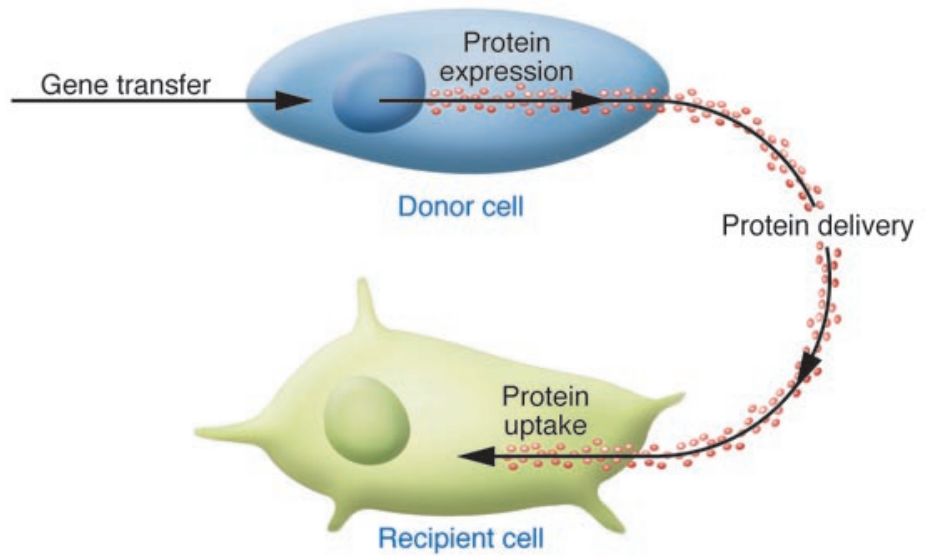

\section{Figure 2}

In vivo versus ex vivo gene therapy. (A) In vivo gene therapy is based on direct gene transfer into the cells requiring the replacement therapy. For CNS diseases, this implies direct injection of vectors into the CNS. (B) For ex vivo gene therapy, the gene transfer is performed in a cell type outside the body (e.g., blood cells), and the genetically modified cells are injected into the deficient recipient. The genetically modified cells can then circulate within the bloodstream, migrate through tissues, and deliver their therapeutic load to neighboring cells - a process known as cross-correction.

phate residues that allow efficient endocytotic uptake of ARSA by neighboring cells via the mannose 6-phosphate receptor. Principally, this pathway could be exploited for cross-correction of ARSA deficiency and to deliver exogenous ARSA derived from normal cells to the lysosomes of ARSA-deficient cells. Accordingly, beginning in 1980, MLD patients have been treated by bone marrow transplantation of allogeneic normal cells in the hope that the circulating normal immune cells cross-correct the deficiency of ARSA. However, the therapeutic potential of bone marrow transplantation is still unclear. It has been suggested that late-onset patients transplanted at presymptomatic stages of disease might profit from this approach (14). But it seems that the amount of ARSA secreted from normal cells after transplantation is insufficient for cross-correcting the deficiency in cells of the patient's CNS. This is surprising since only approximately $5 \%$ of the normal ARSA activity would be sufficient to prevent the manifestation of MLD symptoms (14). Therefore, mice with a deficiency of the murine
Arsa gene, which develop a disorder related to human MLD, have been treated by gene transfer of the human ARSA gene, the gene product of which can functionally replace the missing murine enzyme.

Pioneering work in 2000 by Gieselmann and colleagues showed that retroviral gene transfer in transplanted bone marrow cells is capable of achieving long-term expression of ARSA in the CNS of mice with a deficiency of the murine Arsa enzyme (15). In 2004, Biffi and colleagues published an experimental study in which they transplanted genetically modified hematopoietic bone marrow cells transduced with ARSA into these deficient mice (16). They showed reduced deposition of sulfatide-containing granules after gene therapy and bone marrow transplantation (16). However, this study did not provide evidence for cross-correction of the metabolic defect in oligodendrocytes and did not provide data on ARSA activity in the brain tissue. In this issue of the JCI, Biffi and colleagues have followed up this initial observation and shown, by tagging ARSA with a peptide (HA, derived from the hemaggluti- nin protein of the human influenza virus), that genetically modified and transplanted bone marrow cells can indeed cross-correct deficiency of the enzyme in neighboring brain cells, including neurons and oligodendrocytes in mice (8). Furthermore, they demonstrate that bone marrow transplantation of ARSA-transduced hematopoietic stem and precursor cells increased brain ARSA activity to a level approximately $10 \%$ of that measured in wild-type mice, indicating that therapeutically sufficient levels of ARSA were achieved within the CNS. Finally, the authors show that migration and engraftment of bone marrow-derived myeloid precursors within the CNS are required to achieve correction of the enzyme in Arsa-/- mice, since the transplantation of ARSA-overexpressing hepatocytes increased plasma levels of ARSA but failed to deliver the enzyme to the CNS.

Although the data suggest the potential for a new therapeutic option for MLD patients, several caveats have to be considered. First, it is still unclear whether lentiviral vectors are safe or whether they trigger adverse effects such as leukemogenesis, as previously described for retroviral vectors used in a gene therapy approach in X-linked SCID patients (17). Second, the mouse model, having a deficiency of the murine Arsa enzyme, develops a disease that is closely related to human MLD but is of a generally milder phenotype compared with that observed in MLD patients. These $\mathrm{Arsa}^{-/}$mice do not show widespread and progressive demyelination and may reflect an early stage of MLD (14).

In conclusion, the study by Biffi and colleagues (8) demonstrates in an animal model that genetically modified bone marrow-derived cells overexpressing ARSA migrate into the CNS and can mediate the cross-correction of murine Arsa deficiency in neighboring brain cells. Furthermore, the data suggest that microglia might serve as a natural cellular vehicle for gene therapy of the diseased CNS.

\section{Acknowledgments}

The Neural Regeneration Group at the University of Bonn LIFE \& BRAIN Center is supported by the Hertie Foundation and the Walter and Ilse-Rose Foundation.

Address correspondence to: Harald Neumann, Neural Regeneration Unit, Institute of Reconstructive Neurobiology, University of Bonn, Sigmund-Freud-Strasse 25, 53127 Bonn, Germany. Phone: 49-228-6885-541; Fax: 49-228-6885-501; E-mail: hneuman1@ uni-bonn.de. 
1. Niederkorn, J.Y. 2006. See no evil, hear no evil, do no evil: the lessons of immune privilege. Nat. Immunol. 7:354-359.

2. Pardridge, W.M. 2005. The blood-brain barrier: bottleneck in brain drug development. NeuroRx. 2:3-14

3. Pardridge, W.M. 2002. Drug and gene delivery to the brain: the vascular route. Neuron. 36:555-558.

4. Ghose, A.K., Viswanadhan, V.N., and Wendoloski, J.J. 1999. A knowledge-based approach in designing combinatorial or medicinal chemistry libraries for drug discovery. 1. A qualitative and quantitative characterization of known drug databases. J. Comb. Chem. 1:55-68.

5. Kageyama, T., et al. 2000. The 4F2hc/LAT1 complex transports L-DOPA across the blood-brain barrier. Brain. Res. 879:115-121.

6. Priller, J., et al. 2001. Targeting gene-modified hematopoietic cells to the central nervous system: use of green fluorescent protein uncovers microglial engraftment. Nat. Med. 7:1356-1361.
7. Asheuer, M., et al. 2004. Human CD34+ cells differentiate into microglia and express recombinant therapeutic protein. Proc. Natl. Acad. Sci. U. S. A. 101:3557-3562.

8. Biffi, A., et al. 2006. Gene therapy of metachromatic leukodystrophy reverses neurological damage and deficits in mice. J. Clin. Invest. 116:3070-3082. doi:10.1172/JCI28873.

9. Streit, J.M. 2005. Microglial cells. In Neuroglia. 2nd Edition. Oxford University Press. New York, New York, USA. 60-71.

10. Hickey, W.F., and Kimura, H. 1988. Perivascular microglial cells of the CNS are bone marrow-derived and present antigen in vivo. Science. 239:290-292.

11. Simard, A.R., and Rivest, S. 2004. Bone marrow stem cells have the ability to populate the entire central nervous system into fully differentiated parenchymal microglia. FASEB J. 18:998-1000.

12. Santambrogio, L., et al. 2001. Developmental plasticity of CNS microglia. Proc. Natl. Acad. Sci. U. S. A 98:6295-6300
13. Nimmerjahn, A., Kirchhoff, F., and Helmchen, F. 2005. Resting microglial cells are highly dynamic surveillants of brain parenchyma in vivo. Science. 308:1314-1318.

14. Matzner, U., and Gieselmann, V. 2005. Gene therapy of metachromatic leukodystrophy. Expert Opin. Biol. Ther. 5:55-65.

15. Matzner, U., Harzer, K., Learish, R.D., Barranger, J.A., and Gieselmann, V. 2000. Long-term expression and transfer of arylsulfatase A into brain of arylsulfatase A-deficient mice transplanted with bone marrow expressing the arylsulfatase A cDNA from a retroviral vector. Gene Ther. 7:1250-1257.

16. Biffi, A., et al. 2004. Correction of metachromatic leukodystrophy in the mouse model by transplantation of genetically modified hematopoietic stem cells. J. Clin. Invest. 113:1118-1129. doi:10.1172/ JCI200419205.

17. Hacein-Bey-Abina, S., et al. 2003. LMO2-associated clonal $\mathrm{T}$ cell proliferation in two patients after gene therapy for SCID-X1. Science. 302:415-419.

\title{
Shaping the sperm head: an ER enzyme leaves its mark
}

\author{
Angshumoy Roy, ,1,2 Yi-Nan Lin,, 1,3 and Martin M. Matzuk,2,3 \\ ${ }^{1}$ Department of Pathology, ${ }^{2}$ Department of Molecular and Human Genetics, and \\ ${ }^{3}$ Department of Molecular and Cellular Biology, Baylor College of Medicine, Houston, Texas, USA.
}

\begin{abstract}
Lipid storage diseases are debilitating inherited metabolic disorders that stem from the absence of specific lysosomal enzymes that degrade selected lipids. Most characteristically, these disorders affect the nervous and the reticulo-endothelial systems, with massive organomegaly resulting from the presence of engorged, lipid-laden macrophages. In this issue of the JCI, Yildiz et al. describe the role of the ER-resident enzyme $\beta$-glucosidase 2 (GBA2) in mice (see the related article beginning on page 2985). Surprisingly, GBA2 deficiency leaves bile acid and cholesterol metabolism intact, instead causing lipid accumulation in the ER of testicular Sertoli cells, round-headed sperm (globozoospermia), and impaired male fertility.
\end{abstract}

Glycosphingolipid metabolism in mammalian cells is maintained by a group of synthetic and degradative enzymes that often act in different cell types and subcellular compartments to achieve homeostasis. The term glycosphingolipidoses refers to a group of inherited glycosphingolipid storage disorders in humans (reviewed in ref. 1) caused by mutations in enzymes that degrade specific lipids in the acidic $\mathrm{pH}$ of lysosomes. Clinically, these disorders present with widespread systemic manifestations, with the nervous, reticulo-endothelial, and pul-

Nonstandard abbreviations used: GBA, $\beta$-glucosidase acid; GBA2, $\beta$-glucosidase 2 ; Gopc, golgi-associated PDZ and coiled motif-containing protein; Hrb, HIV-1 Rev-binding protein; $N B-D N J, N$-butyldeoxynojirimycin.

Conflict of interest: The authors have declared that no conflict of interest exists.

Citation for this article: J. Clin. Invest. 116:2860-2863 (2006). doi:10.1172/JCI30221. monary systems severely affected. Since glycosphingolipids are important in cell signaling and development, glycosphingolipidoses are often onset in infancy or childhood and may be fatal if left untreated.

\section{Glycolipids do their bit for posterity}

Several classes of glycolipids are essential for spermatogenesis, as demonstrated by spermatogenic failure in mice lacking either seminolipid or gangliosides (2, 3). Morphologically abnormal spermatozoa and impaired fertility are also seen in a mouse model of the lipid storage disorder Niemann-Pick disease, with lipid accumulation in testicular Sertoli cells (4). In humans, the most common glycosphingolipid storage disorder is Gaucher's disease (OMIM 230800), which is caused by mutations in $\beta$-glucosidase acid (GBA; also known as glucosylceramidase) and results in glucosylceramide accumula- tion in lysosomes. Patients with Gaucher's disease commonly exhibit hepatosplenomegaly and hematological abnormalities from glucosylceramide accumulation, but affected males can sire offspring. Unfortunately, Gba-null mice die perinatally (5), precluding studies on the effects of glucosylceramide accumulation on spermatogenesis (Figure 1). Current therapy for Gaucher's disease involves either recombinant GBA enzyme replacement or administration of $\mathrm{N}$-butyldeoxynojirimycin (NB-DNJ; also known as miglustat), an alkylated iminosugar that reduces glucosylceramide formation by inhibiting UDP-glucose ceramide glucosyltransferase, the resident Golgi apparatus enzyme that transfers glucose moieties to ceramide on the cytosolic face of the early Golgi compartment (6) (Figure 1).

In this issue of the JCI, Yildiz et al. (7) show that the ER enzyme $\beta$-glucosidase 2 (GBA2) is also capable of acting as a glucosylceramidase in vivo in mice - a role hitherto exclusively ascribed to its more familiar lysosomal counterpart, GBA. The authors created a mouse line lacking $G b a 2$ and report that bile acid and cholesterol metabolism as well as protein glycosylation in the testes of Gba2null mice were minimally affected. However, a specific glycolipid - glucosylceramide accumulated in Sertoli cells of Gba2-null testes. Incidentally, in patients with Gaucher's 